\title{
Results From the Swedish National Anterior Cruciate Ligament Register
}

\author{
Joanna Kvist, Juri Kartus, Jon Karlsson and Magnus Forssblad
}

\section{Linköping University Post Print}

\section{Tweet}

N.B.: When citing this work, cite the original article.

Original Publication:

Joanna Kvist, Juri Kartus, Jon Karlsson and Magnus Forssblad, Results From the Swedish National Anterior Cruciate Ligament Register, 2014, Arthroscopy: The Journal of Arthroscopy And Related, (30), 7, 803-810.

http://dx.doi.org/10.1016/j.arthro.2014.02.036

Copyright: WB Saunders

http://www.elsevier.com/

Postprint available at: Linköping University Electronic Press

http://urn.kb.se/resolve?urn=urn:nbn:se:liu:diva-109244 


\section{Results from the Swedish National Anterior Cruciate Ligament}

\section{register.}

Joanna Kvist ${ }^{1}$, P.T., Ph.D., Jüri Kartus ${ }^{2}$, M.D. , Ph.D., Jon Karlsson ${ }^{3}$, M.D., Ph.D., Magnus

Forssblad $^{4}$, M.D., Ph.D.

${ }^{1}$ Department of Medical and Health Sciences, Division of Physiotherapy, Linköping

University, Sweden

${ }^{2}$ NU-Hospital Group, Trollhättan/Uddevalla, Sweden

${ }^{3}$ Department of Orthopaedics, Sahlgrenska University Hospital, Sahlgrenska Academy,

University of Gothenburg, Sweden

${ }^{4}$ Stockholm Sports Trauma Research Center, Karolinska Institutet, and Capio Artro Clinic AB, Sophiahemmet, Stockholm, Sweden

Corresponding author: Joanna Kvist, Department of Medical and Health Sciences, Division of Physiotherapy, Linköping University, SE-581 83 Linköping, Sweden

E-mail: joanna.kvist@liu.se Telephone: +46 13224664 Fax: +46 101031706 


\section{Abstract}

Purpose: The purpose of the study was to report baseline variables and clinical outcome for almost 24,000 patients entered into the Swedish national ACL register.

Methods: The register consists of two parts; one section in which surgeons report baseline and surgical data and one section in which patients report the Knee injury and Osteoarthritis Outcome Score (KOOS) and the EQ-5D before and one, two and five years after surgery.

Results: By December 2012, 23,744 patients had been entered in the surgeons' part of the register. The female/male ratio in the register is $42 / 58$. The mean age at primary $A C L$ reconstruction was 26 (SD 11) and 28 (SD 9) years for the female and male patients respectively. The $A C L$ injury occurred during soccer in $36 \%$ of the females and $49 \%$ of the males. In 2012, 95\% of the primary ACL reconstructions were hamstring tendon autografts. For patients who had a minimum of five years of follow-up, the revision rate was $3.3 \%$ and the contralateral reconstruction rate was $3.8 \%$. On all follow-up occasions up to five years, the patients who had undergone revision had a significantly $(p<0.001)$ poorer outcome than those undergoing primary unilateral $\mathrm{ACL}$ reconstructions in all KOOS and $\mathrm{EQ}-5 \mathrm{D}$ dimensions. On all follow-up occasions, smokers obtained significantly poorer scores than non-smokers in terms of both the KOOS $(p<0.008)$ and the EQ-5D scores $(p<0.024)$. 
Conclusion: Soccer is the most common cause of injury in both females and males, patients undergoing revisions fare less well than those undergoing primary unilateral $\mathrm{ACL}$ reconstructions and smokers fare less well than non-smokers. The cumulative risk of an $\mathrm{ACL}$ revision or contralateral $\mathrm{ACL}$ reconstruction during a five-year period is approximately $7 \%$. For patients under the age of 19 years, the cumulative risk is significantly higher.

Level of Evidence: Level II, Lesser-quality prospective study 


\section{Introduction}

In Scandinavia, there is a long tradition when it comes to disease-specific registers. The hip and knee replacement registers in particular have helped surgeons to abandon less successful products and methods at an early stage. Register studies are useful complements to randomized trials, especially when large sample sizes are needed in order to reveal small yet important differences.

The Swedish National ACL Register (www.aclregister.nu) is a continuous, ongoing study that was initiated in January 2005 and comprises patients undergoing $A C L$ reconstruction and $A C L$ revisions. The register contains both surgeon-reported data at baseline and patient-reported outcomes before the reconstruction and at the one-, two- and five-year follow-ups. Information on smoking has been available since January 2009. The register covers more than $90 \%$ of all ACL procedures performed annually in Sweden and provides important information about the treatment and outcome after ACL injuries. Some results based on data from the register have previously been published. ${ }^{1,2}$ Similar registers exist in other countries, such as the Danish ${ }^{3}$ and Norwegian registers, ${ }^{4}$ as well as the Multicenter Orthopedic Outcomes Network study (MOON study). ${ }^{5}$ In addition, databases like the Kaiser Permanente Anterior Cruciate Ligament Reconstruction Registry (KPACLRR) ${ }^{6}$ and the New York database ${ }^{7}$ exist but without patient-reported outcomes. 
The aim of the present study is to report register findings, including subgroup analyses for primary, revision and bilateral reconstruction, for patients entered between January 2005 and December 2012. Specifically, we hypothesized that young patients suffer an increased risk in undergoing revision reconstruction of the index knee and primary reconstruction of the contralateral knee. Furthermore, those patients who undergo revision reconstruction or bilateral reconstructions would have worse outcomes than patients who undergo unilateral primary reconstruction. 


\section{Methods}

The main functions of the Swedish ACL Register have previously been reported. ${ }^{2}$ The register is a general database that utilizes a web-based protocol. Based on the Swedish social security number, age and gender are registered automatically, and the most recent postal address is continuously updated. The protocol consists of two parts; one in which the surgeons report baseline and surgical data and one with patient-reported outcome measurements (PROM).

In the surgeon-reported section, factors such as activity at injury, time from injury to reconstruction, graft selection and fixation techniques are registered. Previous surgery on the reconstructed knee, the contralateral knee and concomitant injuries are also registered. Revisions and reconstruction on the contralateral knee are registered as separate entries in the database and connected to the primary $\mathrm{ACL}$ reconstruction procedure.

In the PROM section, the patients report the Knee injury and Osteoarthritis Outcome Score $(\mathrm{KOOS})^{8,9}$ and the EQ-5D. ${ }^{10}$ The KOOS is knee specific and covers five domains; symptoms, pain, activities of daily living (ADL), function in sport and recreation (sport/rec) and kneerelated quality of life (QOL). Each subscale score runs from "100 = best" to " $0=$ worst". 
The $E Q-5 D^{10}$ is a non-disease-specific, patient-based evaluation of quality of life consisting of two parts. The first consists of five domains (mobility, self-care, usual activities, pain/discomfort and anxiety/depression), which are converted to a summary index ranging from " 1 = full health" to "-0.594 = worst imaginable health state". The second part is a vertical visual analogue scale (VAS) on which the patients rate their overall health state; "100 $=$ best imaginable health state" and " $0=$ worst imaginable health state". The patient section is reported using the web-based protocol, before the reconstruction, as well as one, two and five years after surgery. The questionnaires together with instructions on how to log in to the web-system are sent to the patients with at least one reminder, for those who don't enter their web-based outcome measures or return their questionnaires within a few months. The patient has the option to fill out the paper forms or log into the web-based system. The web-based protocol includes several drop-down menus. If any answer is left out, the protocol warns that an answer is missing before registration is possible. That secures that there are no questions that are missed to be answered, if the patient chooses to fill out the web-based questionnaires. If the patient chooses not to fill out the protocol at all, in spite of multiple reminders, then the patient is lost to follow-up.

The database complies with the Swedish legislation relating to data security, which means that a non-authorized person can never obtain access to individual data. 


\section{Data analysis and statistical methods}

Patient-related outcomes for those patients that had undergone revision reconstruction or reconstruction of the contralateral knee were followed up for each reconstruction only until the next reconstruction was performed. All subsequent registrations were considered to be related to the latest reconstruction.

Sub-group analyses were done for primary, revision and bilateral reconstruction, smokers and non-smoker, gender and type of graft. Time between injury and reconstruction and between primary and revision reconstruction are reported as median and interquartile range (IQR). Mean values are reported for all the KOOS subscales and EQ-5D values. A one-way ANOVA with Bonferroni post-hoc test was used to compare the KOOS and EQ-5D values between subgroups and the chi-square test was used for dichotomous comparisons. Statistical significance was set at $p<0.05$. 


\section{Results}

By December 2012, 23,744 patients had been entered into the surgeon-related part of the register; 22,059 unique patients had undergone primary $A C L$ reconstruction and 1,685 patients had undergone either multiple ligament reconstructions or revision surgery. Thirtyseven patients had incomplete data, 530 reconstructions were performed on the contralateral knee, i.e. patients had undergone bilateral $\mathrm{ACL}$ reconstructions on different occasions, and 1,431 revision reconstructions had been performed. Of the revision reconstructions, 558 patients underwent revision on a primary reconstruction previously recorded in the register (633, IQR 582, days after primary reconstruction) and 873 revision reconstructions were revisions performed on primary reconstructions performed before 2005 (the start of the register), re-revision or missing values.

Soccer was the most common cause of injury for both males and females. Patient demographics, the time from injury to surgery and the common causes of injury for males and females respectively are given in Table 1. Patients with bilateral reconstructions were two years younger ( $p<0.001$, whole group), (females 3 years, $p=0<001$; males n.s.) compared with the unilateral group at the time of surgery and 2.5 years younger $(p<0.001$, whole group), (females 3 years $p<0.001$; and males 2 years, $p=0.002$ ) compared with the revision group. For each patient group, unilateral, bilateral and revision, $61 \%, 69 \%$ and $52 \%$ respectively were injured in contact sports. The corresponding values for non-contact sports were $20 \%, 15 \%$ and $13 \%$ respectively. Information on smoking was available in 9,332 patients; 579 (6\%) were smokers. 
In 2012, 95\% of the primary ACL reconstructions were performed using hamstring tendon autografts. The graft choice for primary ACL reconstructions over the years is shown in Figure 1. Double-bundle reconstruction was performed in a total of 598 patients. The most common fixation devices in 2005-2012 are listed in Tables $2 a$ and b. Transfixation implants were the most commonly used fixation devices on the femoral side in 2005 , whereas, in 2012, cortical fixation implants were the most commonly used implants on the femoral side, $(p<0.001)$

At the time of surgery, the patients who had undergone revisions had significantly fewer concomitant meniscal injuries (33\% versus $42 \%$ ) but more concomitant chondral injuries ( $44 \%$ versus $27 \%$ ) and more combined meniscal and chondral injuries ( $17 \%$ versus $15 \%$ ) than primary $A C L$ reconstructions ( $p<0.038)$, (Table 3$)$.

The percentages of the maximum possible entries for the KOOS and EQ-5D data, i.e. response rate, preoperatively and at one, two and five years varied between $38 \%$ and $72 \%$ and are shown in Table 4.

The KOOS values for the primary, bilateral and revision reconstructions preoperatively and at one, two and five years are shown in Figures 2a-c. The EQ-5D values for the primary, bilateral and revision reconstructions preoperatively and at one, two and five years are shown in Figures 3a-b. 
On all follow-up occasions, the patients who had undergone revisions had a significantly poorer outcome than those that had undergone primary reconstructions in all KOOS dimensions (differences between 5 and 19 points, $p<0.01$ ), EQ-5D index (differences between 0.067 and 0.101, $p<0.001$ ) and EQ-5D VAS (differences between 6 and $7, p<0.001$ ). Patients with bilateral reconstructions obtained scores similar to those for primary unilateral reconstructions for all KOOS and EQ-5D dimensions on all follow-up occasions, except for KOOS-QoL at the two-year follow-up, where patients undergoing bilateral reconstructions scored 7 points lower than the unilateral group $(p=0.008)$. Patients undergoing bilateral reconstructions obtained higher scores than the revision group on all follow-up occasions on the KOOS subscales for pain, sport/rec and QoL ( 4 to 20 points higher, $p<0.01$ ) and significantly higher at some follow-up points for the KOOS symptom and ADL subscales and the EQ-5D dimensions. Preoperatively, patients undergoing reconstruction for bilateral injuries scored 5 points higher on the sports/rec subscale and 6 points higher on the QoL subscale, compared with those that had unilateral injuries $(p<0.01)$.

Small but significant differences were present on all follow-up occasions, both for the KOOS and EQ-5D scores, mostly favoring the male patients, when comparing females and males undergoing primary unilateral $\mathrm{ACL}$ reconstructions. On all follow-up occasions the females obtained between 4 and 5 points worse scores compared with males only in terms of the sport/rec dimension $(p<0.001)$. For the bilateral and revision groups small or no genderrelated differences were found. 
Except for the preoperative VAS score, significantly worse scores were found in smokers than in non-smokers on all follow-up occasions, both in terms of the KOOS (between 4 and 16 points difference, $p<0.008)$ and the EQ-5D scores $(p<0.024)$ (Figures 4 and 5$)$.

In Figures $6 a$ and $b$ and $7 a$ and $b$, the KOOS and EQ-5D results after using patellar tendon or hamstring tendon autografts for primary reconstructions are presented. Significant, but small differences were found between the groups. However, on all follow-up occasions the patients undergoing reconstruction using hamstring tendon autograft scored between 6-7 points better for the KOOS sports/rec dimension.

When comparing double- versus single-bundle reconstructions using hamstring tendon autografts, the double-bundle reconstructions had slightly lower preoperative scores (2-4 points) in terms of the KOOS symptom, pain and ADL subscales and better (7 points) in terms of the QoL subscale at the five-year follow-up.

Of the patients who underwent primary ACL reconstruction in 2005-2007, 3.3\% (33 of 985 (3.3\%) in the patellar tendon group and 197 of 5,961 (3.4\%) in the hamstring tendon group, n.s.) underwent revision $A C L$ reconstruction of the index knee and 3.8\% underwent $A C L$ reconstruction of their contralateral knee during a minimum five-year postoperative period until December 2012. For patients under the age of 19 at the primary ACL reconstruction, a 
$5.7 \%$ revision rate and $7.1 \%$ bilateral reconstruction rate was found $(p<0.001$, versus the whole cohort). In the whole study group, no gender-related differences in the revision or bilateral reconstruction rate were found. Nine patients $(0.1 \%)$ underwent a second revision of their index knee. 


\section{Discussion}

The principal findings in the present study were that the revision and contralateral reconstruction rates have decreased compared with a previous report from the Swedish register. ${ }^{2}$ Patients who had undergone revision or bilateral $A C L$ reconstruction had a poorer outcome than patients who had undergone primary $\mathrm{ACL}$ reconstruction. Furthermore, smokers fared less well than non-smokers. Gender- and graft-related differences were small and a shift from the use of transfixation devices to cortical fixation devices on the femoral side has occurred. These results are a true representation of the Swedish population who has undergone $A C L$ reconstruction, and most of the information should be generally applicable.

The present study mostly confirms previously reported results from the Swedish ACL Register presented by Ahldén et al. ${ }^{2}$ Two more years of demographics and outcomes, involving almost 6,000 additional patients and analyses of EQ5D data and data from patients with bilateral injuries have now been added. The most important finding is the trend towards lower revision and contralateral reconstruction rates. Unfortunately due to different inclusion criteria, a direct comparison with the results of Ahlden el al. ${ }^{2}$ is not possible. Other studies from registers and databases report a revision rate of between $3 \%$ and $7.7 \%^{6,11-14}$ and a contralateral reconstruction rate of $4.6 \%$ to $6.4 \%$ for a five-year followup period. ${ }^{11,12}$ The reason for the possible reduction in subsequent reconstructions in this population is unclear and further analysis is needed. 
The gender distribution for primary and revision reconstructions is similar to that in the last report from the $A C L$ Register. ${ }^{2}$ From the Danish register, Lind et al. reported a higher male percentage for primary reconstructions (60\%) and a similar gender distribution compared with the present study in terms of revisions. ${ }^{13}$ Other databases report higher female percentages for primary reconstructions ${ }^{11}$ and lower percentages of females with regard to primary and revision reconstructions. ${ }^{6,12}$ Younger patients had a higher rate of revisions and bilateral reconstructions, thereby confirming previous studies. ${ }^{2,6,7,13}$ Previous reports from the Swedish ACL Register have reported a higher rate of re-reconstruction for the specific group of females who sustained their injury playing soccer. ${ }^{2}$ Walden et al. showed that $\mathrm{ACL}$ injuries can be prevented in adolescent female soccer players after introducing an injury prevention program. ${ }^{15}$ Further analyses of age and gender distribution with regard to rereconstructions would be of interest.

Treatment needs to be evaluated from a patient perspective with patient-reported outcomes and treatment choices should be based on the best outcome from a general health-care and patient perspective. The KOOS scores are low at all follow-ups, especially on the subscales for sports and recreation and quality of life. In actual fact, the five-year results after primary $\mathrm{ACL}$ reconstruction in the register are slightly poorer compared with the nonreconstructed group in the randomized study by Frobell et al. ${ }^{16}$ Together with the relatively high risk of graft ruptures and injuries to the contralateral knee, this finding indicates that the treatment after an ACL injury has the potential to be improved. This also indicates that individualized rather than standardized treatment should increase in the future. 
Like previous reports, ${ }^{2,13}$ patients obtained lower scores in terms of the KOOS score after revision surgery. Revision surgery also resulted in a poorer outcome compared with bilateral injuries. It is interesting that patients with bilateral injuries score better on the sport/rec and QoL KOOS subscales before their contralateral reconstruction compared with the preoperative scores for the primary unilateral ACL reconstruction group. Two factors may influence these results; the patients' age and sports participation. Patients undergoing bilateral reconstructions were younger than the other two groups and younger people have been shown to obtain higher scores on the KOOS. ${ }^{1}$ In addition, patients who underwent bilateral reconstructions more frequently sustained their contralateral injury through contact sports compared with patients who underwent primary unilateral reconstruction or revision surgery, indicating that they might have returned to contact sports to a higher degree than the other two groups. Return to sports after primary ACL reconstruction is a risk factor for new injury. Fältström et $\mathrm{al}^{17}$ showed that patients who sustained a contralateral injury after primary ACL reconstruction had a higher activity level before the second injury compared with subjects who did not sustain a contralateral injury. Unfortunately, the register does not provide data on return to sports. One interesting finding is that only $0.1 \%$ of the patients who underwent a primary reconstruction in 2005-2007 underwent a second revision on the same knee. Whether this is due to the fact that the revisions did not re-tear to the same extent as primary reconstruction, or to the fact that the patients did not want to undergo a second revision is an important question. 
In accordance with previous studies of musculoskeletal disorders, ${ }^{2,18}$ smokers obtained lower scores on all PROM evaluation occasions both before reconstruction and at all subsequent follow-ups. Smoking has several harmful effects on general health and on the musculoskeletal system ${ }^{19}$ and smoking cessation reduces postoperative complications. ${ }^{20}$ Data from the register provide an opportunity for further analysis of the effect of smoking on the outcome of $A C L$ reconstruction.

In Sweden, a common treatment algorithm for ACL injured patients is to have patients undergo a supervised rehabilitation period before taking the decision on whether or not a reconstruction is necessary. This is reflected by the time from injury to primary $A C L$ reconstruction, which was a median of more than eight months. This is in line with, but somewhat shorter than, previous reports from the same register. ${ }^{1,2}$

As previously reported, the graft choice in Sweden is predominantly the hamstring tendon autograft. The small differences favoring the hamstring tendon autograft should therefore be interpreted with caution. The comparisons and analyses in terms of double-bundle reconstructions are based on relatively few patients and might therefore be of less value. However, in a randomized study, Ahldén et al. ${ }^{21}$ recently reported no significant differences in clinical and functional outcome when comparing single- and double-bundle $\mathrm{ACL}$ reconstructions. The introduction of the anatomic $A C L$ reconstruction concept and drilling the femoral tunnel through the medial portal is most probably the reason why there has been a shift from transfixation devices to cortical fixation devices on the femoral side. ${ }^{22,23}$ 
The ongoing continuous analysis of data from the national Swedish ACL register is helping to improve and develop treatment algorithms through feedback to hospitals and surgeons with the aim of finding predictive factors for good outcome. ${ }^{24}$ For example, predictive factors related to the lower rate of revisions and contralateral reconstructions should be the focus of future analyses.

\section{Limitations}

National registers provide important data on outcomes and predictors; however, there are some limitations. The register only provides data on new reconstructions and there is only limited knowledge of the actual number of graft failures and injuries to contralateral knees. Another limitation is the relatively low response rate for the PROMs, ranging from $38 \%$ to 72\% on different follow-up occasions. However, a study from the Danish register showed high validity, in spite of the low KOOS response rate. ${ }^{25}$ In addition, no information exists about the rehabilitation. In Sweden, it is common for the patient to participate in a rehabilitation period of at least six months before returning to sports, but we have no information about compliance, which is of course a limitation. 


\section{Conclusion}

Soccer is the most common cause of injury in both females and males, patients undergoing revisions fare less well than those undergoing primary unilateral $A C L$ reconstructions and smokers fare less well than non-smokers. The cumulative risk of a revision or contralateral $\mathrm{ACL}$ reconstruction during a five-year period is approximately $7 \%$. For patients under the age of 19 years, the cumulative risk is significantly higher. 


\section{References}

1.

Ageberg E, Forssblad M, Herbertsson P, Roos EM. Sex differences in patientreported outcomes after anterior cruciate ligament reconstruction: data from the Swedish knee ligament register. Am J Sports Med 2010;38:1334-42.

2. Ahlden M, Samuelsson K, Sernert N, Forssblad M, Karlsson J, Kartus J. The Swedish National Anterior Cruciate Ligament Register: a report on baseline variables and outcomes of surgery for almost 18,000 patients. Am J Sports Med 2012;40:2230-5. 3. Lind M, Menhert F, Pedersen AB. The first results from the Danish ACL reconstruction registry: epidemiologic and 2 year follow-up results from 5,818 knee ligament reconstructions. Knee Surg Sports Traumatol Arthrosc 2009;17:117-24. 4. $\quad$ Granan LP, Forssblad M, Lind M, Engebretsen L. The Scandinavian ACL registries 2004-2007: baseline epidemiology. Acta Orthop 2009;80:563-7.

5. $\quad$ Spindler KP, Parker RD, Andrish JT, et al. Prognosis and predictors of ACL reconstructions using the MOON cohort: a model for comparative effectiveness studies. $J$ Orthop Res 2013;31:2-9.

6. Maletis GB, Inacio MC, Funahashi TT. Analysis of 16,192 Anterior Cruciate Ligament Reconstructions From a Community-Based Registry. Am J Sports Med 2013.

7. $\quad$ Lyman S, Koulouvaris P, Sherman S, Do H, Mandl LA, Marx RG.

Epidemiology of anterior cruciate ligament reconstruction: trends, readmissions, and subsequent knee surgery. J Bone Joint Surg Am 2009;91:2321-8.

$8 . \quad$ Roos EM, Roos HP, Ekdahl C, Lohmander LS. Knee injury and Osteoarthritis Outcome Score (KOOS)--validation of a Swedish version. Scand J Med Sci Sports 1998;8:439-48.

9. Roos EM, Roos HP, Lohmander LS, Ekdahl C, Beynnon BD. Knee Injury and Osteoarthritis Outcome Score (KOOS)--development of a self-administered outcome measure. J Orthop Sports Phys Ther 1998;28:88-96.

10. Brooks R. EuroQol: the current state of play. Health Policy 1996;37:53-72. 11. Hettrich CM, Dunn WR, Reinke EK, Spindler KP. The Rate of Subsequent Surgery and Predictors After Anterior Cruciate Ligament Reconstruction: Two- and 6Year Follow-up Results From a Multicenter Cohort. Am J Sports Med 2013;41:1534-40. 12. Wasserstein D, Khoshbin A, Dwyer T, et al. Risk Factors for Recurrent Anterior Cruciate Ligament Reconstruction: A Population Study in Ontario, Canada, With 5-Year Follow-up. Am J Sports Med 2013.

13. Lind M, Menhert F, Pedersen AB. Incidence and outcome after revision anterior cruciate ligament reconstruction: results from the Danish registry for knee ligament reconstructions. Am J Sports Med 2012;40:1551-7.

14. Rahr-Wagner L, Thillemann TM, Pedersen AB, Lind M. Comparison of Hamstring Tendon and Patellar Tendon Grafts in Anterior Cruciate Ligament Reconstruction in a Nationwide Population-Based Cohort Study: Results From the Danish Registry of Knee Ligament Reconstruction. Am J Sports Med 2013.

15. Walden M, Atroshi I, Magnusson H, Wagner P, Hagglund M. Prevention of acute knee injuries in adolescent female football players: cluster randomised controlled trial. BMJ 2012;344:e3042.

16. Frobell RB, Roos HP, Roos EM, Roemer FW, Ranstam J, Lohmander LS. Treatment for acute anterior cruciate ligament tear: five year outcome of randomised trial. BMJ 2013;346:f232. 
17. Faltstrom A, Hagglund M, Kvist J. Patient- reported Knee Function, Quality of Life, and Activity Level After Bilateral Anterior Cruciate Ligament Injuries. Am J Sports Med 2013;accepted.

18. Sanden B, Forsth P, Michaelsson K. Smokers show less improvement than nonsmokers two years after surgery for lumbar spinal stenosis: a study of 4555 patients from the Swedish spine register. Spine (Phila Pa 1976) 2011;36:1059-64.

19. Lee JJ, Patel R, Biermann JS, Dougherty PJ. The musculoskeletal effects of cigarette smoking. J Bone Joint Surg Am 2013;95:850-9.

20. Mills E, Eyawo O, Lockhart I, Kelly S, Wu P, Ebbert JO. Smoking cessation reduces postoperative complications: a systematic review and meta-analysis. Am J Med 2011;124:144-154 e8.

21. Ahlden M, Sernert N, Karlsson J, Kartus J. A Prospective Randomized Study Comparing Double- and Single-Bundle Techniques for Anterior Cruciate Ligament Reconstruction. Am J Sports Med 2013.

22. Karlsson J, Irrgang JJ, van Eck CF, Samuelsson K, Mejia HA, Fu FH. Anatomic single- and double-bundle anterior cruciate ligament reconstruction, part 2: clinical application of surgical technique. Am J Sports Med 2011;39:2016-26.

23. Samuelsson K, Andersson D, Ahlden M, Fu FH, Musahl V, Karlsson J. Trends in surgeon preferences on anterior cruciate ligament reconstructive techniques. Clin Sports Med 2013;32:111-26.

24. $\quad$ Engebretsen L, Forssblad M. Why knee ligament registries are important. Knee Surg Sports Traumatol Arthrosc 2009;17:115-6.

25. Rahr-Wagner L, Thillemann TM, Lind MC, Pedersen AB. Validation of 14,500 operated knees registered in the Danish Knee Ligament Reconstruction Register: registration completeness and validity of key variables. Clin Epidemiol 2013;5:219-28. 26. Frobell RB, Svensson E, Gothrick M, Roos EM. Self-reported activity level and knee function in amateur football players: the influence of age, gender, history of knee injury and level of competition. Knee Surg Sports Traumatol Arthrosc 2008;16:7139. 


\section{Figure legends}

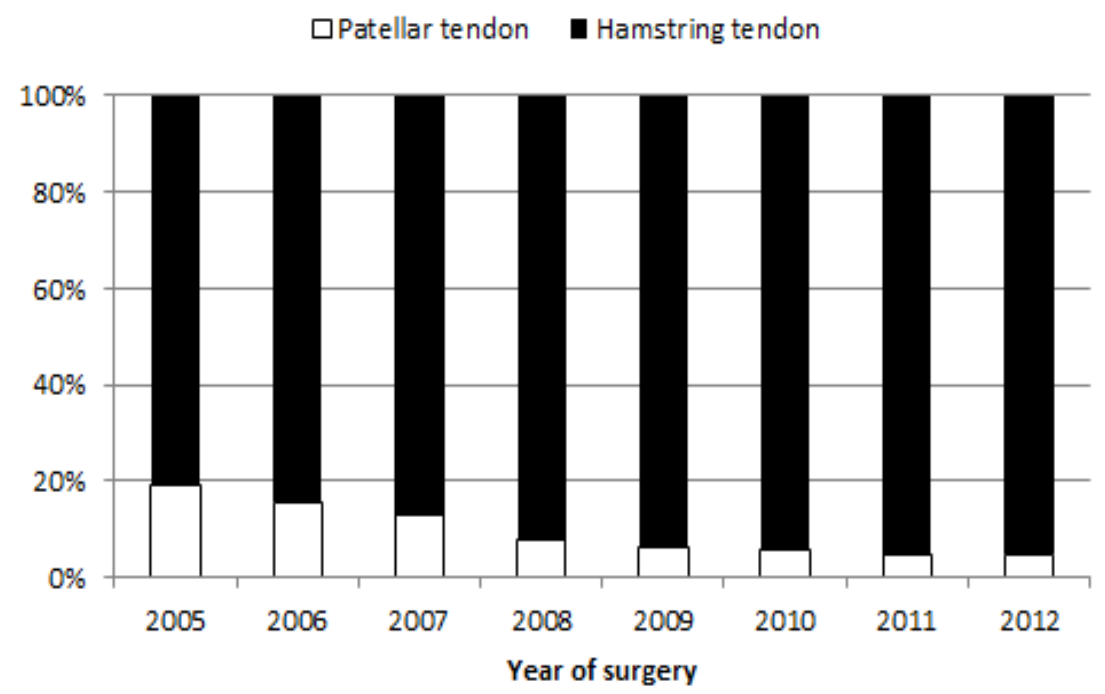

Figure 1. Graft choice over time for primary ACL reconstruction.

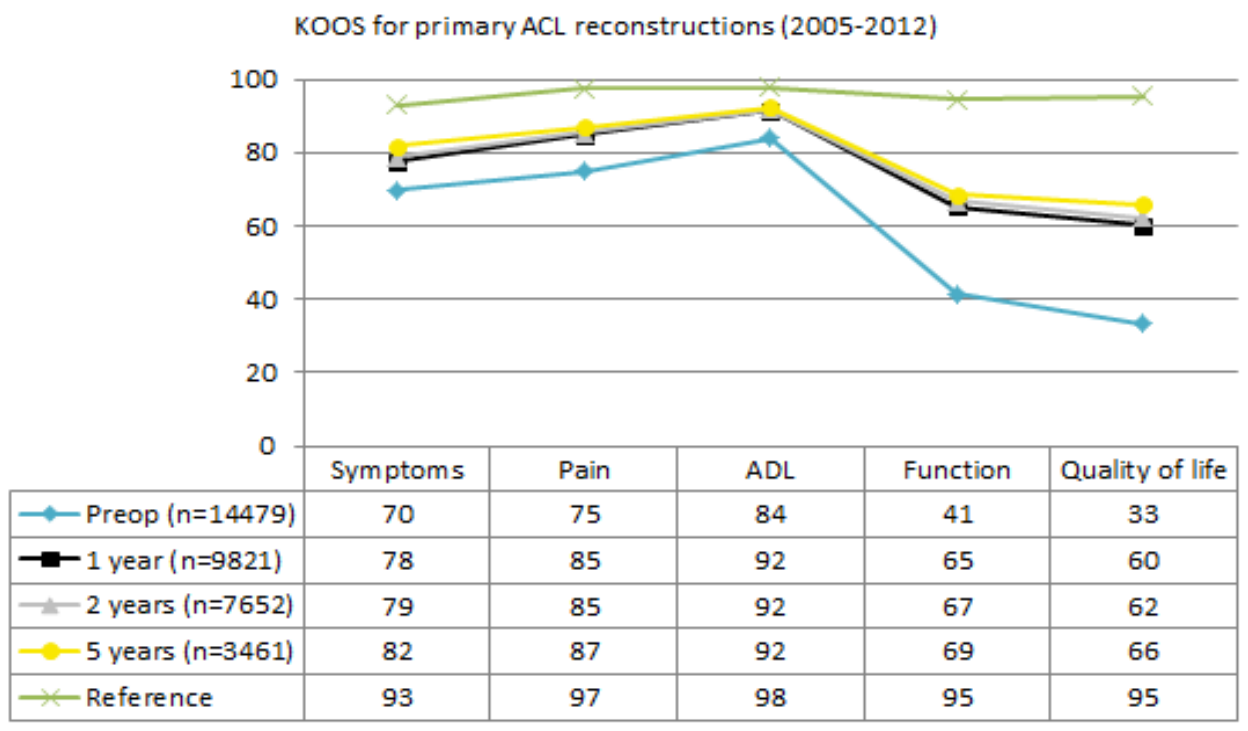



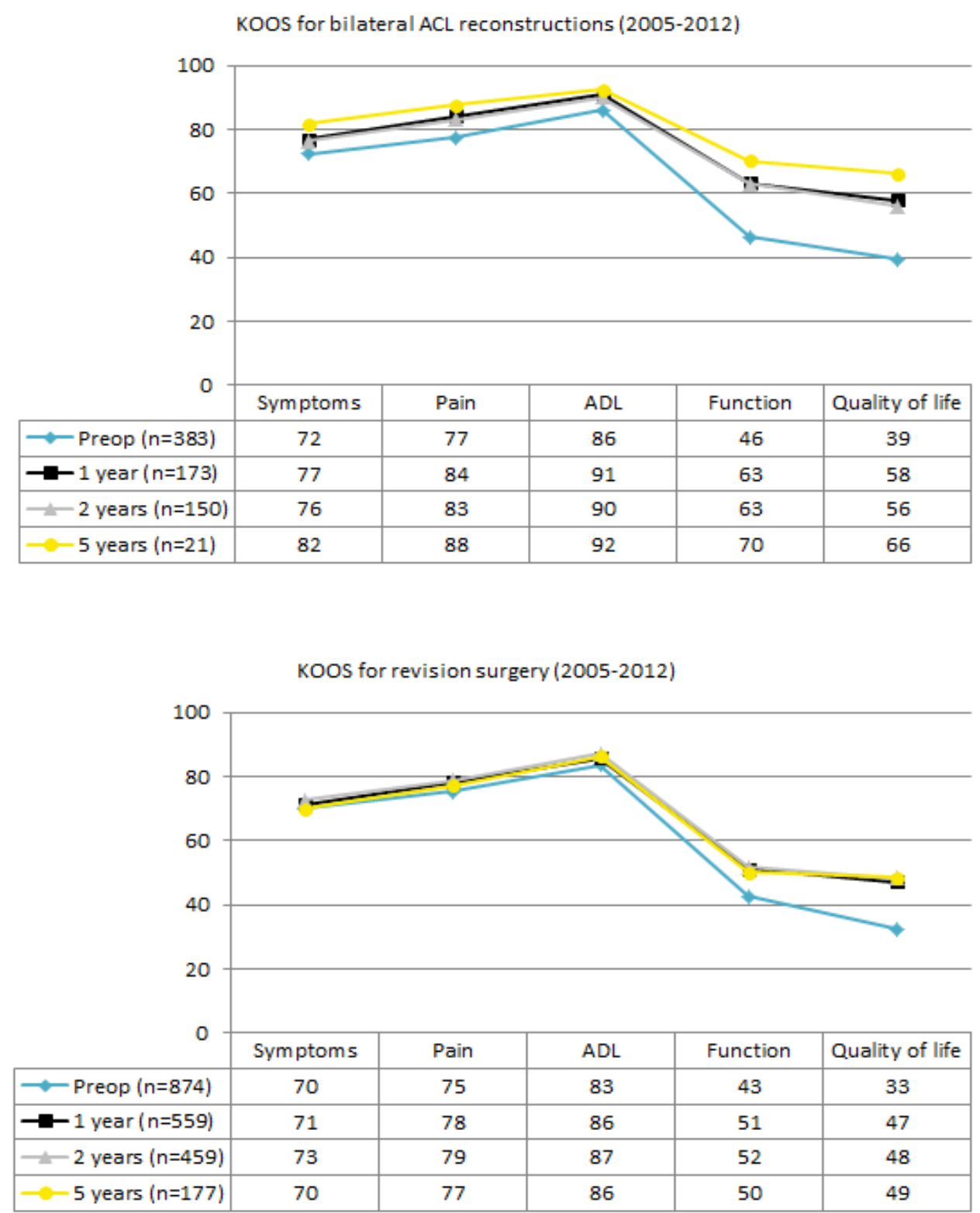

Figure 2. Mean values for KOOS for primary (2a), bilateral (2b) and revision (2c)

reconstructions. Reference values are from 118 knee-healthy soccer players with no history of knee injury. ${ }^{26}$ 

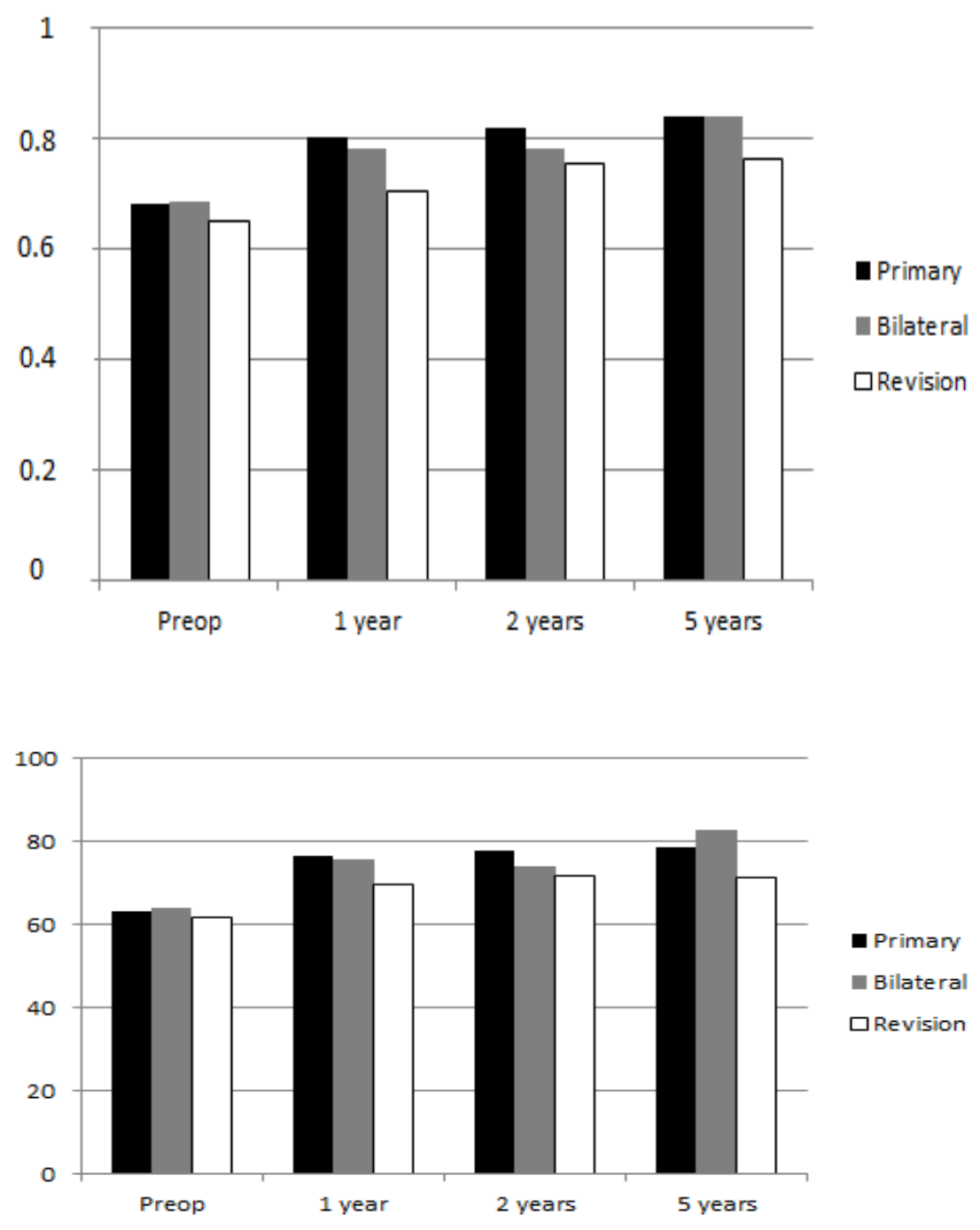

Figure 3. Mean values for EQ-5D index (3a) and VAS (3b) for primary, bilateral and revision reconstructions. 

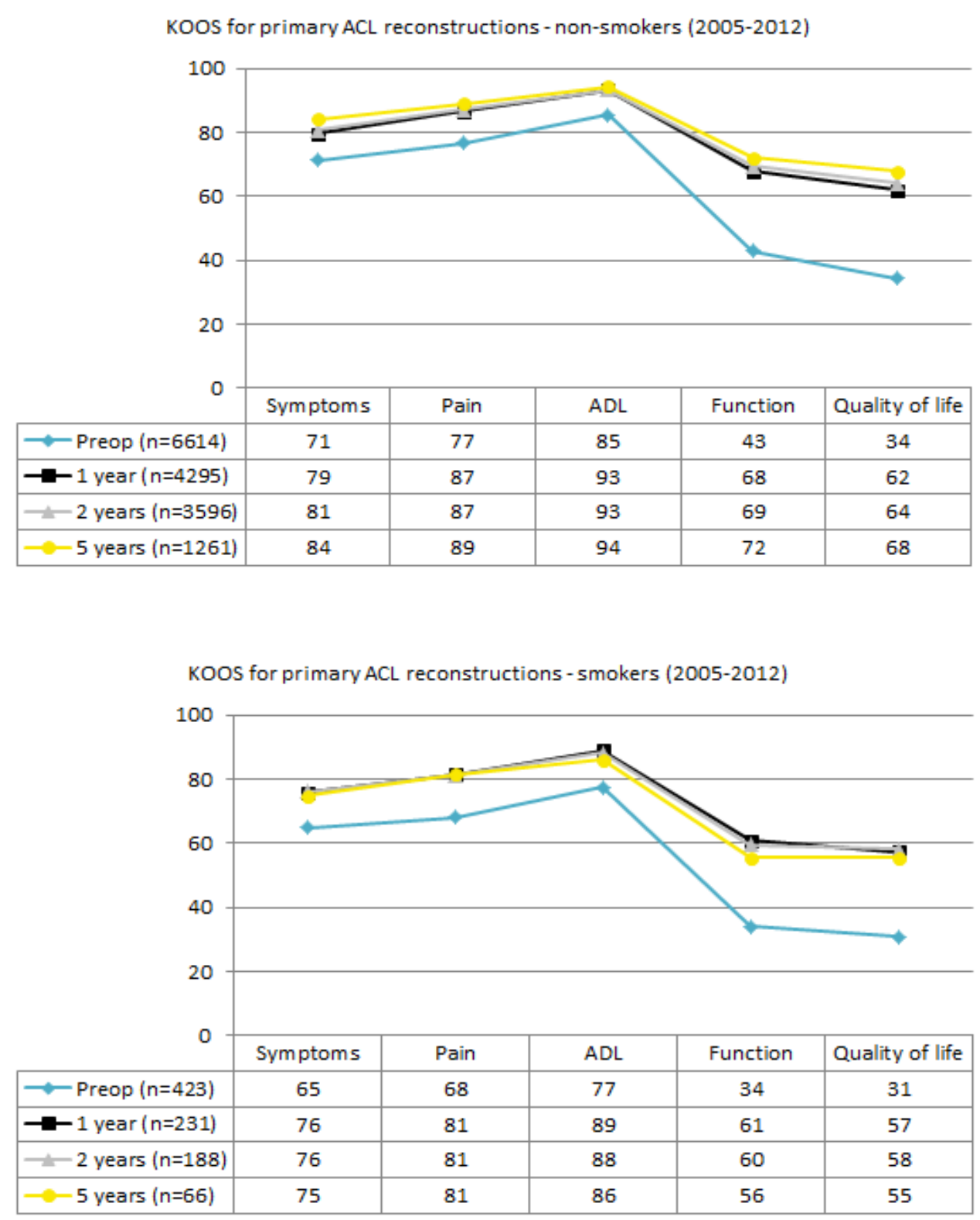

Figure 4. Mean values for KOOS for non-smokers (4a) and smokers (4b). 


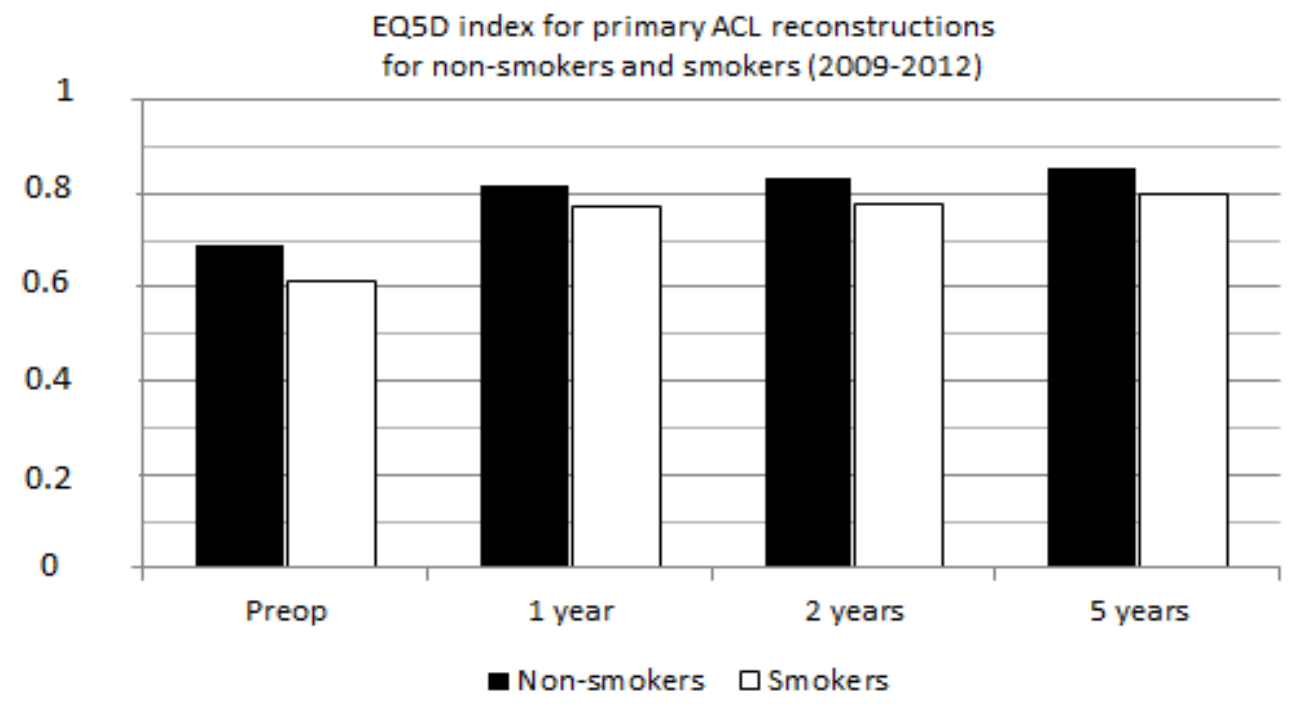

EQ-VAS for primary $\mathrm{ACL}$ reconstructions

for non-smokers and smokers (2009-2012)

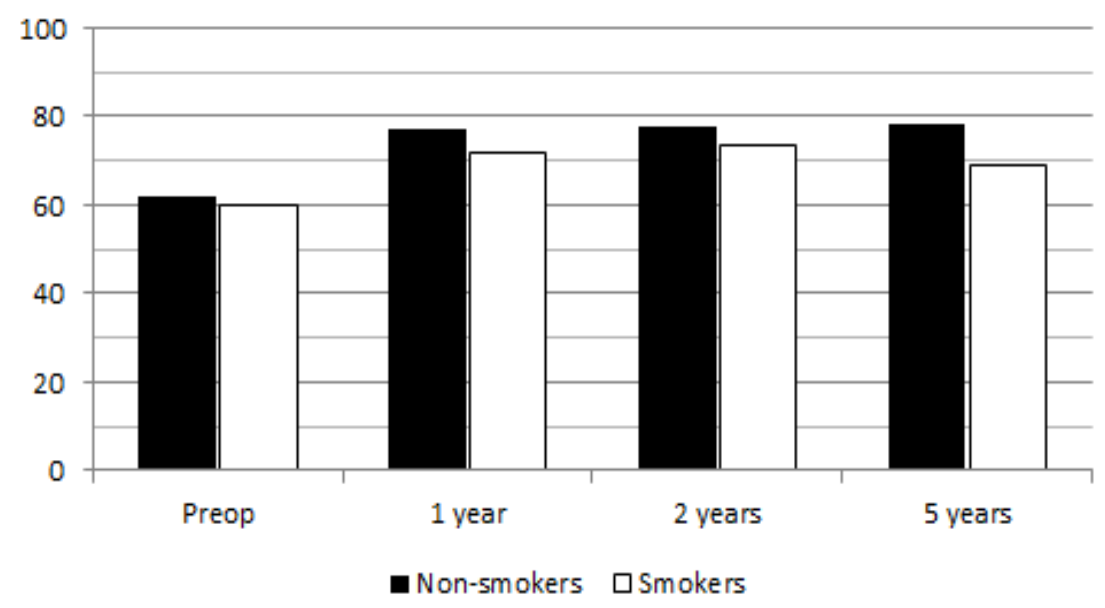

Figure 5. Mean values for EQ-5D index (5a) and VAS (5b) for non-smokers and smokers respectively. 
KOOS for primary ACL reconstructions; patellar tendons (2005-2012)

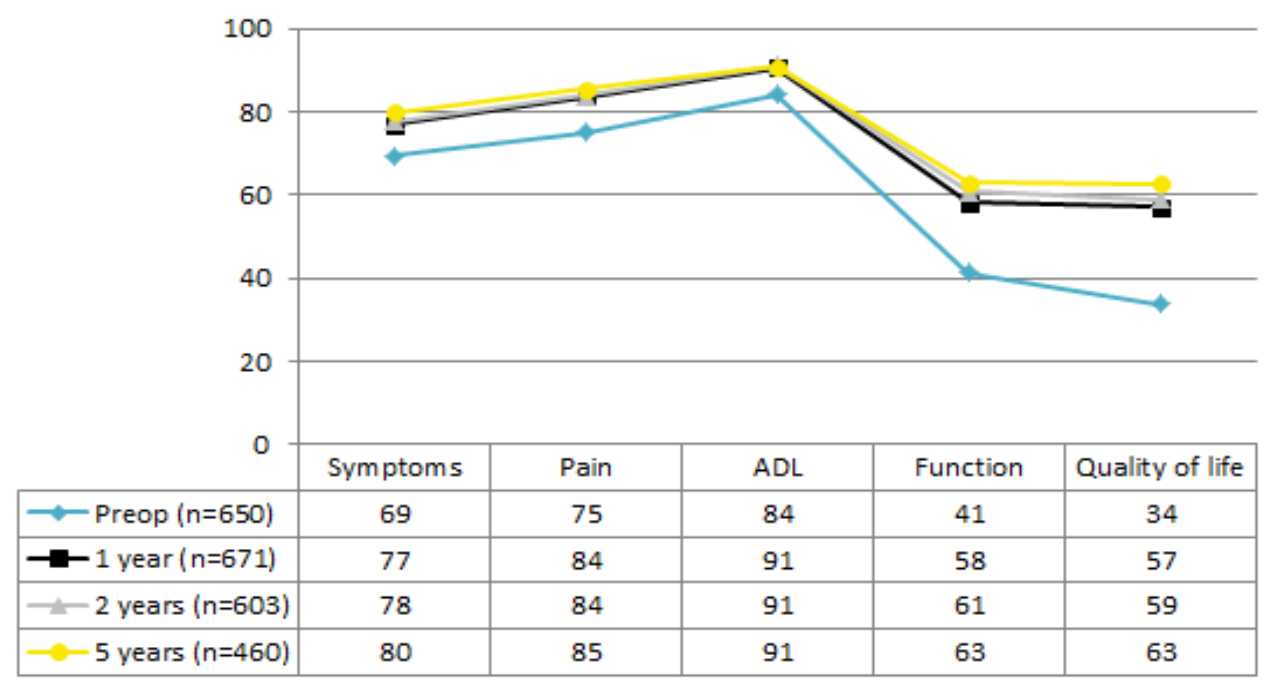

KOOS for primary ACL-reconstructions; hamstring tendons (2005-2012)

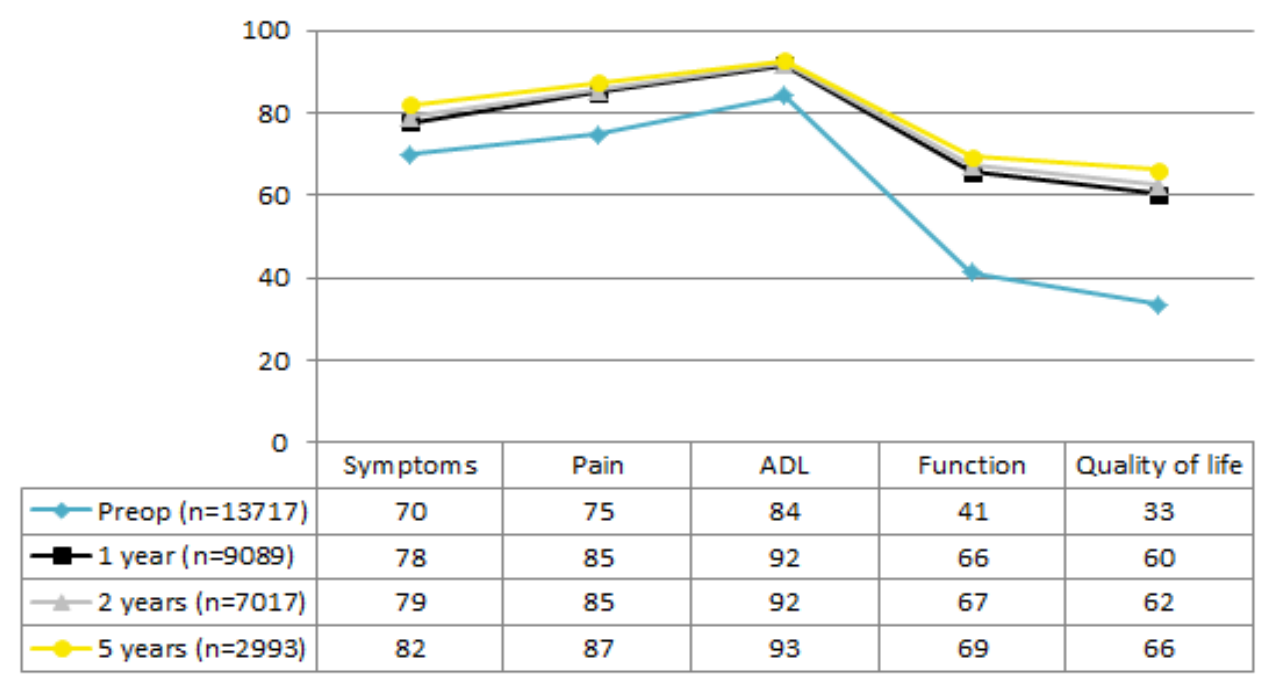

Figure 6. Mean values for KOOS for patellar tendon (6a) and hamstring tendon (6b) autografts in patients undergoing primary $A C L$ reconstructions. 
EQ5D index for primary ACL reconstructions by graft (2005-2012)

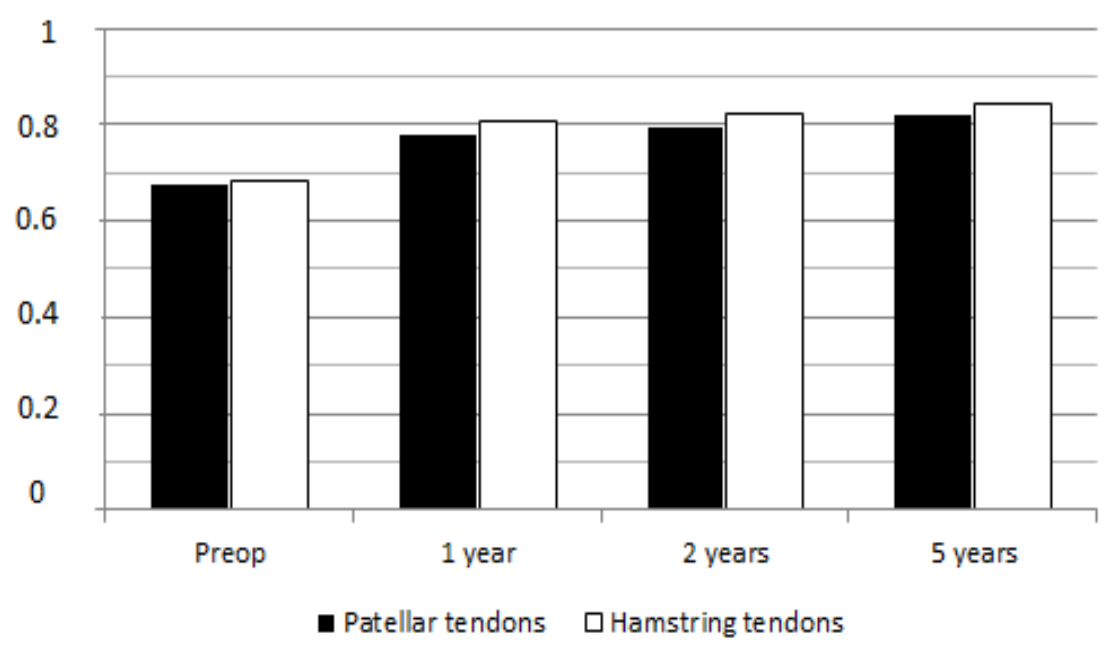

EQ-VAS for primary ACL reconstructions by graft (2005-2012)

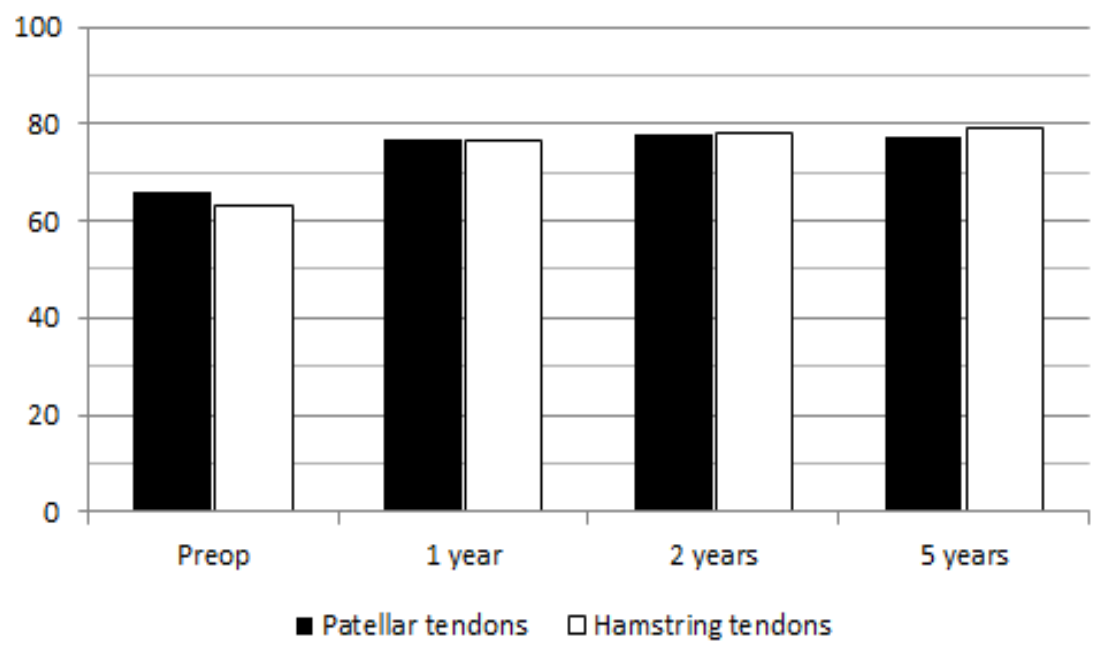

Figure 7. Mean values for EQ-5D index (7a) and VAS (7b) for patients undergoing ACL reconstruction using patellar and hamstring tendon autografts. 
Table 1. Demographics for primary, bilateral and revision reconstructions

\begin{tabular}{|l|l|l|l|l|l|l|l|l|l|}
\hline & \multicolumn{3}{|c|}{ Primary } & \multicolumn{3}{c|}{ Bilateral } & \multicolumn{3}{c|}{ Revision } \\
\hline & Female & Male & Total & Female & Male & Total & Female & Male & Total \\
\hline $\mathrm{N}$ & $\begin{array}{l}9332 \\
(42 \%)\end{array}$ & $\begin{array}{l}12727 \\
(58 \%)\end{array}$ & 22059 & $\begin{array}{l}246 \\
(46 \%)\end{array}$ & $\begin{array}{l}284 \\
(54 \%)\end{array}$ & 530 & $\begin{array}{l}624 \\
(44 \%)\end{array}$ & $\begin{array}{l}807 \\
(56 \%)\end{array}$ & 1431 \\
\hline Age at surgery, mean (SD) & $26(11)$ & $28(9)$ & $27(10)$ & $23(9)$ & $27(8)$ & $25(8)$ & $26(9)$ & $29(8)$ & $28(9)$ \\
\hline $\begin{array}{l}\text { Time from injury to surgery } \\
\text { in days, median (IQR) }\end{array}$ & $\begin{array}{l}251 \\
(347)\end{array}$ & $\begin{array}{l}266 \\
(382)\end{array}$ & $\begin{array}{l}260 \\
(367)\end{array}$ & $\begin{array}{l}153 \\
(187)\end{array}$ & $\begin{array}{l}177 \\
(233)\end{array}$ & $\begin{array}{l}162 \\
(217)\end{array}$ & NA** $^{* *}$ & NA $^{* *}$ & NA $^{* *}$ \\
\hline Most common cause $\left(^{*}\right)$ & & & & & & & & & \\
\hline Soccer & $36 \%$ & $49 \%$ & $43 \%$ & $45 \%$ & $53 \%$ & $49 \%$ & $34 \%$ & $45 \%$ & $40 \%$ \\
\hline $\begin{array}{l}\text { Alpine skiing (incl. } \\
\text { snowboard) }\end{array}$ & $18 \%$ & $10 \%$ & $14 \%$ & $10 \%$ & $7 \%$ & $9 \%$ & $11 \%$ & $6 \%$ & $8 \%$ \\
\hline European handball & $9 \%$ & $3 \%$ & $6 \%$ & $13 \%$ & $4 \%$ & $8 \%$ & $6 \%$ & $3 \%$ & $4 \%$ \\
\hline Floorball & $8 \%$ & $10 \%$ & $9 \%$ & $9 \%$ & $9 \%$ & $9 \%$ & $5 \%$ & $5 \%$ & $5 \%$ \\
\hline
\end{tabular}

SD: standard deviation, IQR: interquartile range

* Percentage of total injuries in each group ** Uncertain data in the register 
Table 2. Fixation Devices, Year by Year, on Tibial and Femoral Sides for Primary ACL Reconstructions Using Hamstring Tendon Autografts

\begin{tabular}{|c|c|c|c|c|c|c|c|c|c|}
\hline & 2005 & 2006 & 2007 & 2008 & 2009 & 2010 & 2011 & 2012 & Total \\
\hline \multicolumn{10}{|l|}{ Tibial side (\%) } \\
\hline Metal screw & 41.6 & 39.8 & 39.9 & 32.3 & 34.9 & 34.6 & 32.6 & 24.8 & 34.4 \\
\hline Intrafix (DePuy Mitek, Raynham, MA) & 26.9 & 25.4 & 20.0 & 20.1 & 13.1 & 9.4 & 8.0 & 7.9 & 15.3 \\
\hline Absorbable screw & 0.0 & 0.3 & 4.3 & 13.5 & 14.8 & 19.6 & 24.3 & 32.4 & 15.2 \\
\hline Suture Post & 7.6 & 7.1 & 9.5 & 12.4 & 16.8 & 16.9 & 15.9 & 17.1 & 13.5 \\
\hline Metal screw + suture post fixation & 10.8 & 13.8 & 10.8 & 9.8 & 13.0 & 13.6 & 14.1 & 13.7 & 12.6 \\
\hline Rigidfix (DePuy Mitek) & 6.5 & 7.5 & 7.3 & 5.6 & 3.1 & 2.2 & 0.9 & 0.9 & 3.9 \\
\hline RetroScrew (Arthrex, Naples, FL) & 1.8 & 3.0 & 4.7 & 3.3 & 2.1 & 2.6 & 1.7 & 0.6 & 2.4 \\
\hline Other & 4.6 & 2.8 & 3.0 & 2.6 & 1.9 & 0.7 & .8 & 0.9 & 2.0 \\
\hline $\begin{array}{l}\text { EndoButton/RetroButton/TightRope (Smith } \delta \\
\text { Nephew, Andover, MA/Arthrex/Arthrex) }\end{array}$ & 0.1 & 0.2 & 0.5 & 0.5 & 0.3 & 0.3 & 1.8 & 1.6 & 0.7 \\
\hline \multicolumn{10}{|l|}{ Femoral side $(\%)$} \\
\hline EndoButton/RetroButton/TightRope & 9.8 & 18.1 & 22.7 & 34.6 & 46.0 & 59.2 & 69.0 & 79.0 & 45.7 \\
\hline Rigidfix/TransFix (Arthrex) & 60.4 & 55.2 & 53.4 & 43.9 & 32.0 & 18.8 & 11.0 & 5.3 & 32.2 \\
\hline Metal screw & 28.7 & 26.2 & 23.1 & 20.4 & 19.9 & 19.6 & 16.9 & 13.5 & 20.4 \\
\hline Other & 1.0 & 0.4 & 0.8 & 1.1 & 2.1 & 2.4 & 3.0 & 2.2 & 1.8 \\
\hline
\end{tabular}


Table 3. Associated injuries in primary and revision surgery

\begin{tabular}{|l|l|l|l|}
\hline & Primary & Revision & $\mathrm{P}$ \\
\hline Meniscal injury & $42 \%$ & $33 \%$ & $<0.001$ \\
\hline Chondral injury & $27 \%$ & $44 \%$ & $<0.001$ \\
\hline Meniscal and chondral injury & $15 \%$ & $17 \%$ & 0.038 \\
\hline
\end{tabular}


Table 4. Response rate for KOOS and EQ-5D

\begin{tabular}{|l|l|l|l|l|l|l|}
\hline & \multicolumn{2}{|c|}{ Primary } & \multicolumn{2}{c|}{ Bilateral } & \multicolumn{2}{c|}{ Revision } \\
\hline & KOOS & EQ-5D & KOOS & EQ-5D & KOOS & EQ-5D \\
\hline Pre op & $66 \%$ & $61 \%$ & $72 \%$ & $68 \%$ & $61 \%$ & $58 \%$ \\
\hline 1 year & $50 \%$ & $49 \%$ & $40 \%$ & $38 \%$ & $45 \%$ & $43 \%$ \\
\hline 2 year & $48 \%$ & $47 \%$ & $47 \%$ & $46 \%$ & $45 \%$ & $44 \%$ \\
\hline 5 year & $48 \%$ & $46 \%$ & $38 \%$ & $38 \%$ & $42 \%$ & $42 \%$ \\
\hline
\end{tabular}

